\title{
Pharmacologic and genetic approaches define human pancreatic $\beta$ cell mitogenic targets of DYRK1A inhibitors
}

\author{
Courtney Ackeifi, ${ }^{1}$ Ethan Swartz, ${ }^{1}$ Kunal Kumar, ${ }^{2,3}$ Hongtao Liu, ${ }^{1}$ Suebsuwong Chalada, ${ }^{2,3}$ \\ Esra Karakose, ${ }^{1}$ Donald K. Scott, ${ }^{1}$ Adolfo Garcia-Ocaña, ${ }^{1}$ Roberto Sanchez, ${ }^{2,3}$ Robert J. DeVita, ${ }^{2,3}$ \\ Andrew F. Stewart, ${ }^{1}$ and Peng Wang ${ }^{1}$ \\ 'Diabetes Obesity Metabolism Institute, ${ }^{2}$ Drug Discovery Institute, and ${ }^{3}$ Department of Pharmacological Sciences, Icahn \\ School of Medicine at Mount Sinai, New York, New York, USA.
}

\begin{abstract}
Small molecule inhibitors of dual specificity, tyrosine phosphorylation-regulated kinase $1 \mathrm{~A}$ (DYRK1A), including harmine and others, are able to drive human $\beta$ cell regeneration. While DYRK1A is certainly a target of this class, whether it is the only or the most important target is uncertain. Here, we employ a combined pharmacologic and genetic approach to refine the potential mitogenic targets of the DYRK1A inhibitor family in human islets. A combination of human $\beta$ cell RNA sequencing, DYRK1A inhibitor kinome screens, pharmacologic inhibitors, and targeted silencing of candidate genes confirms that DYRK1A is a central target. Surprisingly, however, DYRK1B also proves to be an important target: silencing DYRK1A results in an increase in DYRK1B. Simultaneous silencing of both DYRK1A and DYRK1B yields greater $\beta$ cell proliferation than silencing either individually. Importantly, other potential kinases, such as the CLK and the CSK3 families, are excluded as important harmine targets. Finally, we describe adenoviruses that are able to silence up to 7 targets simultaneously. Collectively, we report that inhibition of both DYRK1A and DYRK1B is required for induction of maximal rates of human $\beta$ cell proliferation, and we provide clarity for future efforts in structure-based drug design for human $\beta$ cell regenerative drugs.
\end{abstract}

Authorship note: $\mathrm{CA}, \mathrm{ES}$, and KK contributed equally.

Conflict of interest: The Icahn Schoo of Medicine at Mount Sinai has filed patents on DYRK1A inhibitor drugs (no. W0 2018/081401-A1).

Copyright: (ㄷ) 2020, American Society for Clinical Investigation.

Submitted: August 13, 2019

Accepted: December 4, 2019

Published: January 16, 2020.

Reference information: /CI Insight. 2020:5(1):e132594.

https://doi.org/10.1172/jci.

insight.132594.

\section{Introduction}

Diabetes results from inadequate numbers of normally functioning insulin-producing pancreatic $\beta$ cells (1-4). This has prompted attempts to induce residual $\beta$ cells in people with Type 1 diabetes (T1D) and $\mathrm{T} 2 \mathrm{D}$ to replicate or regenerate. In the past 4 years, several groups have shown that drugs that inhibit the $\beta$ cell kinase, dual specificity tyrosine phosphorylation-regulated kinase 1A (DYRK1A) are able to induce proliferation of human $\beta$ cells in vitro and in vivo. This class of human $\beta$ cell proliferation-enhancing DYRK1A inhibitors includes harmine, INDY, leucettine-41, GNF4877, 5-iodotubericidin (5-IT), TG003, AZ191, CC-401, and more recently synthesized DYRK1A inhibitors (5-13). Several reports have shown that the human $\beta$ cell proliferative activity of this class can be mimicked by silencing DYRK1A and can be inhibited by overexpression of DYRK1A in human $\beta$ cells (5-7), making it clear that DYRK1A is an essential mediator of the proliferative response to these drugs.

On the other hand, evidence suggests that there are likely other targets of the DYRK1A inhibitors that participate in induction of human $\beta$ cell proliferation. First, kinome screens performed by multiple groups reveal that each of these DYRK1A inhibitors also inhibit other kinases, particularly members of the CMGC (cyclin-dependent kinase [CDK], mitogen-activated protein [MAP] kinase, glycogen synthase kinase-3 [GSK3], and CDC-like kinase [CLK]) classes - notably DYRK1B, DYRK2, DYRK3, DYRK4, CLK1, CLK2, CLK4, GSK3 $\alpha$, GSK3 $\beta$, and casein kinases (CSNK) 1A, 1D, and E (7-13). Each of these could, in theory, participate in human $\beta$ cell proliferation. GSK3 merits special mention here, since genetic or pharmacologic interference with GSK3 $\beta$ in mice has been reported to lead to rodent $\beta$ cell proliferation $(14,15)$, and Shen et al. have suggested that GSK3 $\beta$ inhibition may contribute to the efficacy of GNF4877 (8). On the other hand, only limited data have been reported in humans. For example, Liu et al. have reported that the GSK3 $\beta$ inhibitors, $\mathrm{LiCl}$ and 1-Akp, increases human $\beta$ cell Ki67 immunolabeling from $0.17 \%$ to $0.71 \%$ (15). Second, dose-response curves for each DYRK1A inhibitor reveal peak rates of human $\beta$ cell 
proliferation at a given dose - after which, proliferation declines (5, 7-13); these are findings that imply detrimental interaction with other kinases or other targets (so-called antitargets) at higher doses. Third, off-target effects need not be restricted to protein kinases. For example, 5-IT is also an adenosine kinase inhibitor; indeed, the $\beta$ cell mitogenic capability of 5-IT was originally attributed to inhibition of adenosine kinase $(7,16)$. Fourth, it is possible that DYRK1A inhibitors may affect targets other than kinases. As an example, harmine not only inhibits DYRK1A in human $\beta$ cells, but it also reduces abundance of SMAD proteins, via uncertain mechanisms (6). As another example, harmine is a monoamine oxidase (MAO) inhibitor (5). Finally, it is clear that the mitogenic effects of DYRK1A inhibitors operate via translocation of NFaT transcription factors to the nucleus, with the consequent transactivation of cyclins, such as cyclin $\mathrm{A}$, and CDKs, such as CDK1, and repression of CDK-inhibitors such as p15 $5^{\mathrm{INK} 4}, \mathrm{p} 21^{\mathrm{CIP} 1}$, and p57 $7^{\mathrm{KIP} 2}(5,8$, 11, 16-19). On the other hand, additional effects are likely: DYRK1A phosphorylates and stabilizes p2 $7^{\mathrm{KIP}}$ (11); phosphorylates D-cyclins, accelerating their degradation (20); phosphorylates the DREAM complex member, LIN52, enforcing cell cycle arrest (21); and phosphorylates the Alzheimer's disease protein, Tau (22). Collectively, while these observations make it clear that DYRK1A inhibition is an important mechanism underlying human $\beta$ cell proliferation induced by harmine and related DYRK1A inhibitors, they also illustrate that other potential kinase and nonkinase targets exist that may enhance and/or restrict human $\beta$ cell mitogenic activity, and that these additional targets vary, depending on the particular DYRK1A inhibitor being studied. Deepening this knowledge base is critical to the design and synthesis of future, more potent and selective human $\beta$ cell mitogenic drugs.

With this background, we undertook a detailed exploration of the mechanisms of action and targets of the DYRK1A inhibitor class of small molecules, including harmine, INDY, leucettine-41, GNF4877, 5-IT, CC-401, TG003, and AZ191, on human $\beta$ cell proliferation. We confirm that DYRK1A is unequivocally the principal human $\beta$ cell mitogenic target. Surprisingly, however, we found that $\beta$ cell DYRK1B is an important target, as well. In addition, while GNF4877 and 5-IT are more potent drivers of human $\beta$ cell proliferation than the other members of the group, and are clearly acting in large part or entirely via inhibition of DYRK1A and DYRK1B, kinome scans reveal an exceptionally broad range of potential additional mitogenic and antimitogenic targets for 5-IT and GNF4877. Finally, in order to execute these studies, we developed a useful method for silencing up to 7 CMGC family genes simultaneously in a single target cell by incorporating multiple shRNAs within a single adenovirus. This tool and concept will be valuable to the biological research community in a broad range of future studies.

\section{Results}

Kinome scans and RNA sequencing. To identify potential mitogenic targets of harmine, we performed a kinase inhibitor screen of harmine at a dose of $10 \mu \mathrm{M}$ against 468 human kinases using the DiscoverX platform (Supplemental Table 1; supplemental material available online with this article; https://doi.org/10.1172/ jci.insight.132594DS1). The "hits" showing the greatest inhibition were DYRK1A and CLK1, but other kinases in the CMGC class were also identified, including DYRK1B, DYRK2, CLK1, and CLK2, as were the non-CMGC kinases CSNK1D and CSNK1E. We next explored the abundance of these kinases in human $\beta$ cells via our RNA sequencing (RNA-seq) database of FACS-sorted human $\beta$ cell (Table 1 and refs. 23-25) and found that, in human $\beta$ cells, each of the DYRK, CLK, and GSK3 family members is present, as are CSNK1A, CSNK1D, and CSNK1E, all in comparable abundance with other important kinases such as PI3 kinase, MAP kinase, and Jun kinase. Since harmine is a MAO inhibitor (5), we also queried MAOA and MAOB, and we found them to be only marginally detectable in human $\beta$ cells.

Pharmacologic approaches. We next developed a panel of small molecule inhibitors of the potential DYRK1A inhibitor targets in Table 1 and tested their ability to induce human $\beta$ cells to replicate, as assessed by insulin and Ki67 coimmunolabeling of dispersed cadaveric human islets treated with these drugs. Figure 1 displays the potential mitogenic targets of harmine from the kinome screen and $\beta$ cell RNA-seq across the top row. It also compares an array of DYRK, CLK, GSK3, MAO, and CSNK inhibitors and their reported pharmacologic inhibitory potency and specificity as a heatmap, with green indicating strong inhibitory potency, yellow indicating intermediate potency, and red indicating poor potency. Black boxes indicate either that the drug has no activity against that kinase or that no information is available for that drug versus the kinase in question.

A review of Figure 1 makes the following points. First, drugs that elicit the greatest human $\beta$ cell mitogenic potency are reported to be inhibitors of DYRK1A, DYRK1B, DYRK2, CLK1, CLK4, 
Table 1. Expression of potential harmine targets in human FACS-purified $\beta$ cells

\begin{tabular}{|c|c|}
\hline Gene & FPKM \\
\hline Name & (Average) \\
\hline Insulin & 49184 \\
\hline IAPP & 1029 \\
\hline CGA & 734 \\
\hline CGB & 765 \\
\hline Glucagon & 1261 \\
\hline Somatostatin & 2445 \\
\hline Gamma Actin & 762 \\
\hline Tubulin A1A & 395 \\
\hline PIK3CA & 4 \\
\hline MAPK1 & 12 \\
\hline МАPK8 & 6 \\
\hline DYRIK1A & 5 \\
\hline DYRIK1B & 10 \\
\hline DYRK2 & 6 \\
\hline DYRK3 & 5 \\
\hline DYRK4 & 2 \\
\hline CLK1 & 34 \\
\hline CLK2 & 14 \\
\hline CLIK3 & 3 \\
\hline CLIK4 & 4 \\
\hline GSK3a & 13 \\
\hline GSIK3b & 19 \\
\hline MAOA & 3 \\
\hline MAOB & 1 \\
\hline CSNIK1A & 22 \\
\hline CSNK1D & 21 \\
\hline CSNIK1E & 17 \\
\hline
\end{tabular}

RNA-seq-derived gene expression data from 25 sets of FACS-purified human $\beta$ cells. The data are a composite of RNA-seq from $\beta$ cells labeled with adenovirus encoding RIP-ZsGreen (23) or Newport green (24), or immunolabeling of permeabilized fixed islet cells for insulin (25). The first 8 genes are included to illustrate their level of expression in FACS-sorted human $\beta$ cells and provide a frame of reference for the expression levels of the genes that follow. Note that DYRK family members are present, with DYRK 4 being the least highly expressed. CLKs 1 and 2 are most abundant in this family. GSK3 $\alpha$ and $-\beta$ are also abundant, as are casein kinases $1 A,-1 D$, and $-1 E$. All of these are present at levels similar to PI3 kinase (PIK3CA), MAP kinase (MAKP1), and JUN kinase (MAPK8). Monoamine oxidases, which are wellrecognized targets of harmine in other cell types, are almost undetectable inhuman $\beta$ cells. Data are expressed as mean fragments expressed per kilobase of transcript per million mapped reads (FPKM). Complete data are available in ref. 23 and dbGaP (http://www.ncbi.nlm.nih.gov/projects/gap/cgi-bin/about.html) under accession number phs001422.v1.p1.

MAOA, and MAOB. Several are also weaker inhibitors of CSNK1D and -E. Most are poor inhibitors of GSK $3 \alpha$ and GSK3 $\beta$. Second, harmaline - a close harmine structural analogue and an MAOA/ MAOB inhibitor, but a poor DYRK1A inhibitor - displays no mitogenic efficacy (5). Third, the GSK3 inhibitors, CHIR99021 and tideglusib, fail to induce human $\beta$ cell proliferation (vide infra). Collectively, these observations support 3 high-level interpretations. First, MAOs and GSK3 kinases are unlikely to be relevant to the ability of harmine to induce human $\beta$ cell proliferation. This is evident from the observations that MAOs are not abundant in human $\beta$ cells; MAO inhibitors such as harmaline do not induce proliferation (5); and GSK3 inhibitors do not induce human $\beta$ cell proliferation. Second, no small molecule inhibitor is perfectly and uniquely specific for its nominal target (for example, all available DYRK1A inhibitors for which data are available are also CLK inhibitors). And third, because of this target overlap and since complete kinome scans and/or kinase inhibition assays of many kinase inhibitors in Figure 2 have not been reported, it is impossible - using a purely pharmacologic approach — to precisely and unequivocally define which of the kinase candidates contributes to the mitogenic effects of harmine. 


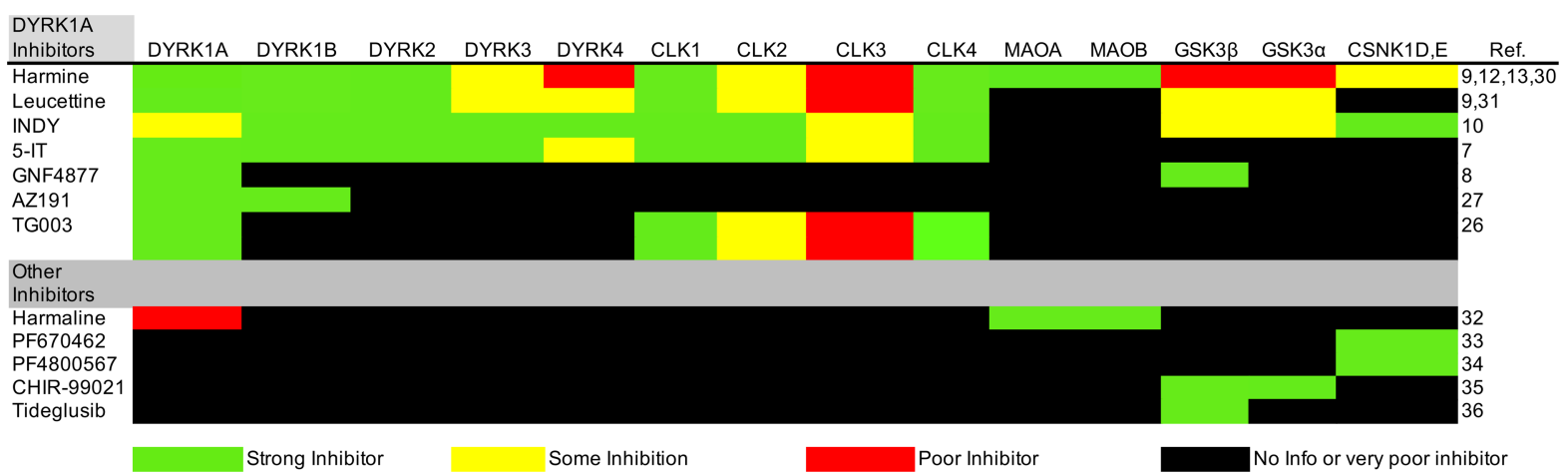

Figure 1. A pharmacologic approach to defining human $\beta$ cell mitogenic targets of the harmine analogue class. This heatmap displays the most likely potential harmine targets derived from the kinome scans described in Table 1 and Supplemental Table 1 along the top row. Column 1 lists the kinase inhibitor drugs employed arranged in 2 clusters. The top group was selected because they are known inhibitors of DYRK1A. The drugs in the bottom group are not DYRK1A inhibitors, but they inhibit other kinases highlighted in the kinome screen in Table 1 and Supplemental Table 1. The color codes are indicated at the bottom of the heatmap. The sources of the data are shown in the references listed in the far right column; in some cases, these data are based in kinase inhibition assays, and in some cases, data are based on percent inhibition of binding in kinome screens, as indicated in the references listed. Taken together, the data make it clear that: (a) no DYRK1A inhibitor is entirely specific for any of the kinases shown, (b) all DYRK1A inhibitors are also DYRK1B inhibitors where data are available, and (c) it is impossible to precisely and unequivocally define relevant human $\beta$ cell mitogenic targets for harmine, INDY, leucettine, 5-IT, and GNF4877 exclusively through the use of small molecule pharmacologic inhibitors (refs. 7-10, 12, 13, 26, 27, 30-36).

Silencing of lead candidate kinases in human islets reveals both DYRK1A and DYRK1B as key mitogenic targets of harmine. Lacking more precise pharmacologic tools, we turned to a genetic approach, building a collection of adenoviruses capable of silencing each of the members of the DYRK family in human islets and observing effects on proliferation. Adenoviruses expressing shRNAs against human DYRK1A, DYRK1B, DYRK2, DYRK3, and DYRK4 were prepared, and each was shown to be effective at reducing expression of their targets by quantitative PCR (qPCR) of the cognate RNA and, where antisera are available, their cognate protein, as assessed by immunoblot (Figure 2, A and B). Remarkably, DYRK1B protein actually increased in immunoblots of human islets when DYRK1A was silenced (Figure 2B), although DYRK1B mRNA did not (Figure 2C). Assessment of proliferation by insulin-Ki67 coimmunolabeling in dispersed human islets confirmed, as reported previously (5-7), that silencing DYRK1A does induce human $\beta$ cells to proliferate (Figure 2D). In contrast, silencing DYRK2, DYRK3, and DYRK4 had no effect on proliferation. Silencing DYRK1B suggested a very small, nonsignificant increase in proliferation.

With the increase in DYRK1B when DYRK1A is silenced in mind, and with the suggestion that silencing DYRK1B leads to a small, nonsignificant increase in human $\beta$ cell proliferation (Figure 2D), we explored whether simultaneous silencing of multiple targets using multiple adenoviruses in Figure 2, A-D - such as DYRK1A, DYRK1B, and other DYRKs or CLKs - might synergize to drive higher rates of human $\beta$ cell proliferation. Unfortunately, simultaneous treatment of human islets with multiple individual adenoviruses expressing DYRK family shRNAs proved toxic to human $\beta$ cells.

As an alternate approach, we therefore prepared single adenoviruses expressing multiple shRNAs directed against all 5 DYRK family members in various combinations, exemplified in Figure 2E. Figure 2F demonstrates that simultaneous silencing of all 5 DYRK family members using a single virus was effective in reducing expression of the cognate gene by an average of $\sim 70 \%$. We also prepared variants of this virus expressing shRNAs directed against only DYRK1A and DYRK1B, or DYRK1A + DYRK1B + DYRK2, or all of the 5 DYRKs including DYRK3 or DYRK4, and we compared their efficacy on inducing human $\beta$ cell proliferation. As expected, silencing DYRK1A induced human $\beta$ cell proliferation. Remarkably, and unexpectedly, simultaneous combined silencing of both DYRK1A and DYRK1B yielded statistically significantly higher rates of proliferation than silencing either alone (Figure 2G). Adding shRNAs directed against DYRK2, DYRK3, and DYRK4 to DYRK1A + DYRKB all resulted in proliferation, but these rates were lower as compared with silencing only DYRK1A and DYRK1B, a result we attribute to interference with other presumably important but unknown functions of the less-studied DYRK family members, DYRKs 2, 3, and 4.

Collectively, these findings support 4 important concepts. First, interfering with DYRK1A activity is central to induction of human $\beta$ cell proliferation by harmine and related DYRK1A inhibitor drugs. Second, inhibition of DYRK1A results in an unexpected, large, and presumably compensatory increase in DYRK1B (Figure 2B). Third, as a result, simultaneous silencing of both DYRK1A and DYRK1B yields a 

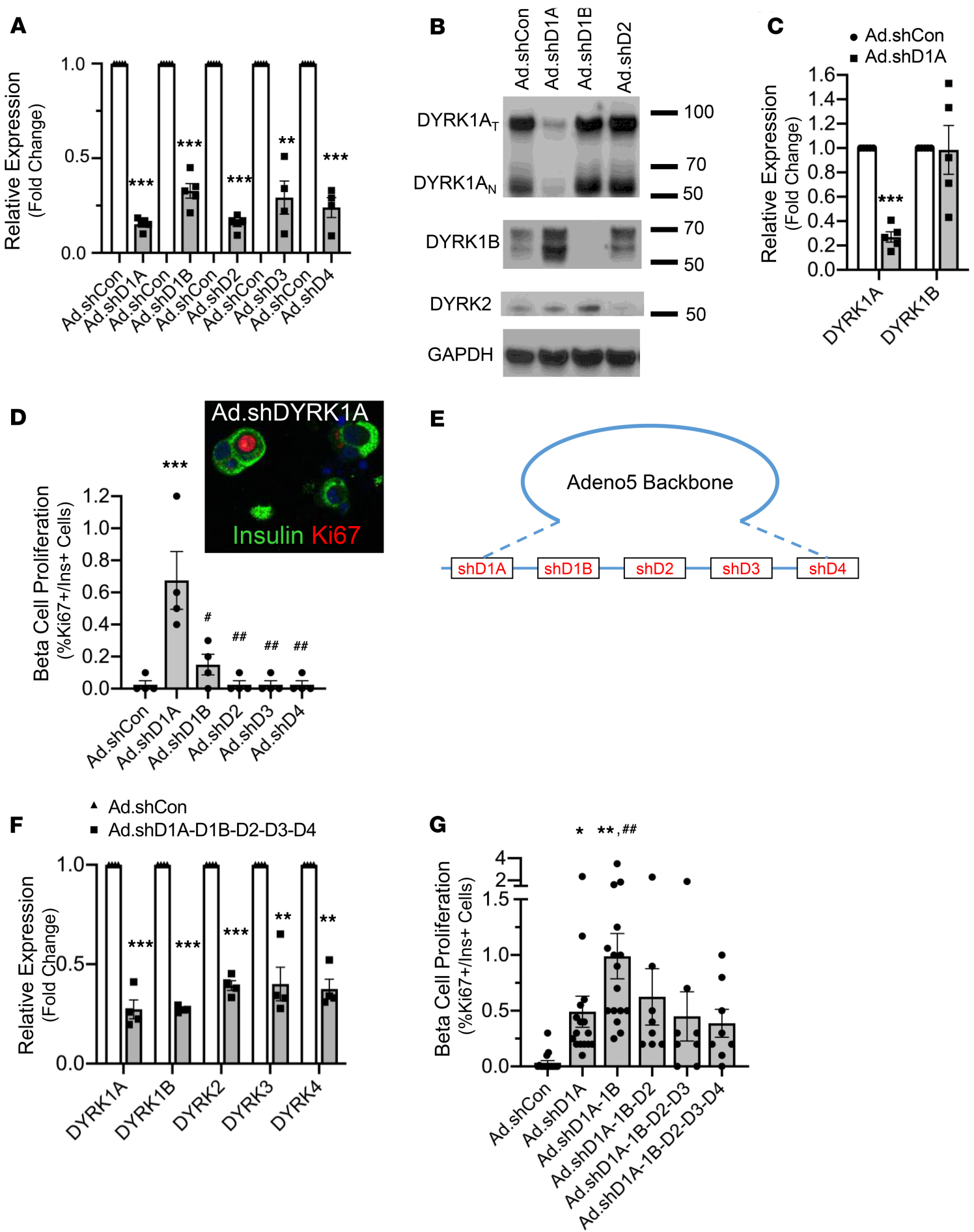

Figure 2. Silencing DYRK1A and DYRK1B in combination induces and enhances human $\boldsymbol{\beta}$ cell proliferation. (A) qPCR studies demonstrating that adenoviruses intended to silence the DYRK family are effective in human islets. Ad.shCon refers to a control adenovirus-silencing $\beta$-galactosidase. Data are shown as the mean \pm SEM of 4 human islet donors. ${ }^{* *} P<0.01$; ${ }^{* * *} P<0.001$ vs. the Ad. shCon control by paired 2 -tailed $t$ test. (B) Immunoblots for the DYRK1A, DYRK1B, and DYRK2 in human islets transduced with the viruses shown. DYRK3 and DYRK4 were not examined because antisera are not available. Each blot represents immunoblots from 3 different donors. The key points are that silencing DYRK1A, DYRK1B, and DYRK2 in human islets is effective, and that silencing DYRK1A leads to an apparent compensatory increase in DYRK1B. (C) qPCR for DYRK1B in response to Ad.shDYRK1A. Data are shown as mean \pm SEM of 5 human islet donors. ${ }^{* *} P<0.001$ vs. the Ad. shCon control by paired 2 -tailed $t$ test. (D) The effects on proliferation (Ki67-insulin coimmunolabeling) of silencing DYRK1A (D1A), DYRK1B (D1B), DYRK2 (D2), DYRK3 (D3), or DYRK4 (D4) individually in human islets. Data are shown as mean \pm SEM of 4 human islet donors. The inset shows a representative example of Ki67 and

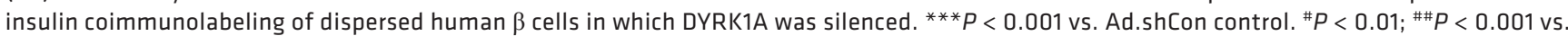
Ad.shD1A, all by 1-way ANOVA with Bonferroni's multiple-comparisons test. Original magnification, 40x. (E) Illustration of shRNAs directed against each of the 5 members of the DYRK family. Other viruses silencing only DYRK1A and DYRK1B, and other combinations shown in the next panels, 
were also generated. (F) qPCR studies demonstrating that a single virus containing shRNA cassettes directed against each member of the DYRK family effectively silences its cognate RNA. Data are shown as mean \pm SEM of 4 human islet donors. ${ }^{* *} P<0.01 ;{ }^{* * *} P<0.001$ vs. the Ad.shCon control by paired 2-tailed $t$ test. (G) Effect on human $\beta$ cell proliferation of a variety of combinations of DYRK shRNA adenoviruses in human $\beta$ cells.

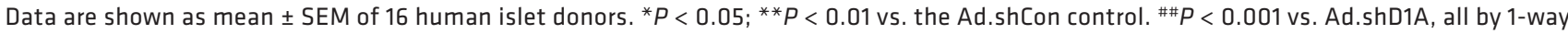
ANOVA with Bonferroni's multiple-comparisons test. Specific human islet preparations used are detailed in Supplemental Table 2.

significant and synergistic further increase in proliferation beyond that observed with silencing DYRK1A or DYRK1B alone (Figure 2G). Fourth, since all DYRK1A inhibitors are also DYRK1B inhibitors (Figure 1 and Supplemental Table 1), it is likely that combined inhibition of DYRK1A and DYRK1B underlies the proliferation of all drugs in the DYRK inhibitor family.

Silencing of GSK3 $\alpha$ and GSK3B, alone or in combination with DYRK1A and DYRK1B, has no effect on human $\beta$ cell proliferation. We next turned our attention to the GSK3 family, preparing adenoviruses that silence GSK $3 \alpha$ and GSK3 $\beta$ individually. Silencing these 2 kinases individually was effective, as assessed by qPCR and immunoblot of human islets (Figure 3, A and B). With regard to proliferation, silencing DYRK1A with the Ad.shDYRK1A in human islets served as a positive control, inducing proliferation in the $0.7 \%$ range. In contrast, silencing of either GSK3 $\alpha$ or GSK3 $\beta$ individually failed to induce $\beta$ cell proliferation (Figure 3C), reminiscent of results with pharmacologic GSK3 $\alpha$ and GSK3 $\beta$ inhibitors $(14,15)$.

Since it remained plausible that GSK inhibition might complement or augment DYRK1A silencingmediated proliferation, we prepared a single adenovirus that simultaneously silences both DYRK1A and DYRK1B, as well as an adenovirus that silences DYRK1A, DYRK1B, GSK3 $\alpha$, and GSK3 $\beta$ (Figure 3, D and E). We observed that silencing both GSK3s in combination with DYRK1A and DYRK1B was no more effective in inducing proliferation than simultaneously silencing DYRK1A and DYRK1B (Figure 3F). These observations are concordant with the pharmacologic inhibition data in Figure 1 and collectively suggest that GSK3 $\alpha$ and GSK3 $\beta$ inhibition does not contribute to, nor augment, proliferation driven by harmine.

Silencing of CLK family members alone or in combination with DYRK1A and DYRK1B has no effect on human $\beta$ cell proliferation. We next turned our attention to the CLK family. Silencing CLKs 1, 2, and 4 individually (Figure 4A) was effective, as assessed by the reduction of their respective gene expression levels. We did not pursue CLK3 because silencing CLK3 alone had no effect on proliferation in preliminary studies and because CLK3 is not inhibited by harmine (Figure 1 and Supplemental Table 1; refs. 12, 13). Unlike DYRKs and GSK3s in Figures 2 and 3, specific antisera effective for immunoblotting CLK family members are not available. Silencing each of the 3 CLKs individually had no effect on human $\beta$ cell proliferation (Figure 4B). To explore the possibility that CLK inhibition may synergize with DYRK1A/B inhibition to augment $\beta$ cell proliferation, we prepared a single adenovirus able to silence DYRK1A, DYRK1B, CLK1, CLK2, and CLK4 (Figure 4, C and D). As can be seen in Figure 4E, simultaneous silencing of DYRK1A, DYRK1B, and CLKs 1, 2, and 4 yielded no additional proliferation beyond that induced by the DYRK1A and DYRK1B combination. Collectively, together with the findings in Figure 1, these observations indicate that, while CLKs may be inhibited by harmine analogs, CLK inhibition does not likely participate in the proliferation induced by harmine.

Comparison of human $\beta$ cell mitogenic potency across the DYRK1A inhibitor family. GNF4877 and 5-IT have been suggested to be more effective than harmine in driving human $\beta$ cell replication $(7,8)$. However, a comprehensive assessment of relative human $\beta$ cell proliferation efficacy by multiple members of the DYRK1A inhibitor family - at multiple doses in large numbers of human islet donors - has not been reported previously. Accordingly, we selected 5 canonical human islet regenerative DYRK1A inhibitors (harmine, INDY, 5-IT, leucettine-41, and GNF4877) (5-10, 11-13) and explored their mitogenic efficacy in the same 10 human islet preparations, using a broad range of doses (Figure 5, A-C). Harmine, INDY, and leucettine-41 yielded comparable dose-response curves and $\mathrm{EC}_{50}$ for proliferation $(\sim 5 \mu \mathrm{M})$, along with similar maximal degrees of proliferation ( 3.5\%). In contrast, the curves for both GNF4877 and 5-IT were shifted to the left $\left(\mathrm{EC}_{50}\right.$ of $\left.\sim 0.5 \mu \mathrm{M}\right)$, indicating that these 2 compounds are about 10 -fold more potent as human $\beta$ cell mitogens than harmine, leucettine-41, or INDY.

We next explored the potency of 3 other putative DYRK1A inhibitors (TG003, AZ191, and CC-401) $(11,26,27)$ as human $\beta$ cell mitogens in different sets of human islets, again with harmine as a control (Figure 5, D and E). Among this group, AZ191 was inferior with regard to maximal proliferation efficacy as compared with harmine, INDY, Leucettine-41, 5-IT, and GNF4877. AZ191 also caused $\beta$ cells to shrink and detach from tissue culture slides, suggesting that it is toxic to $\beta$ cells at the doses studied. 
A

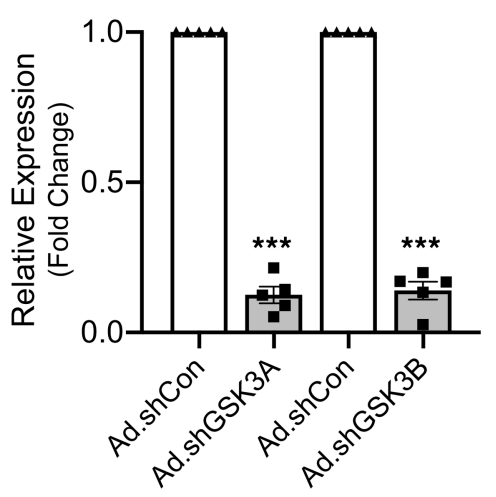

D

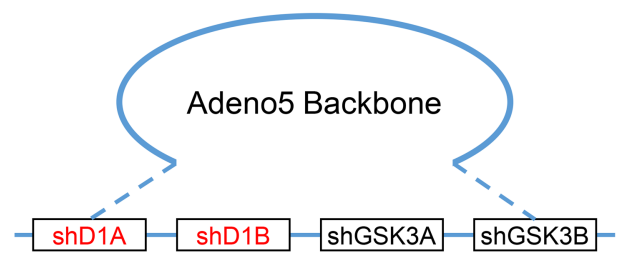

B

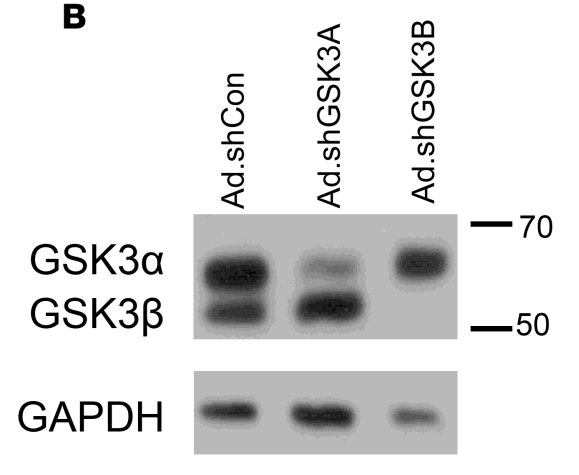

- Ad.shCon

- Ad.shD1A-1B-GSK3A-3B

E

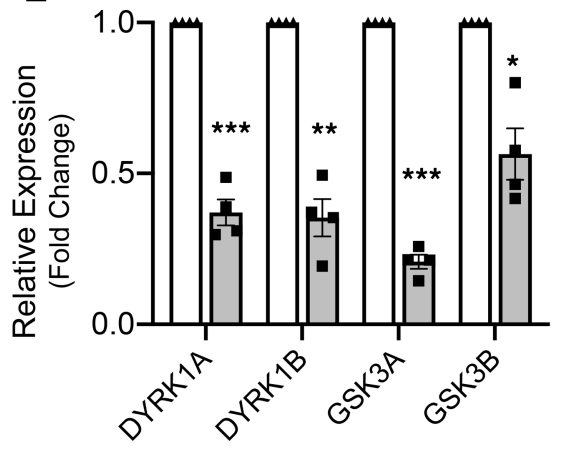

C

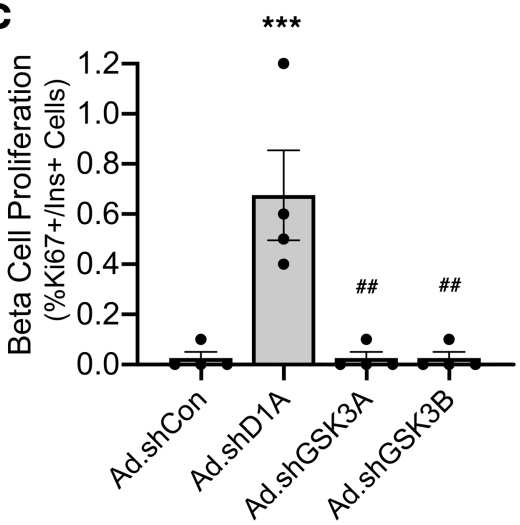

$\mathbf{F}$

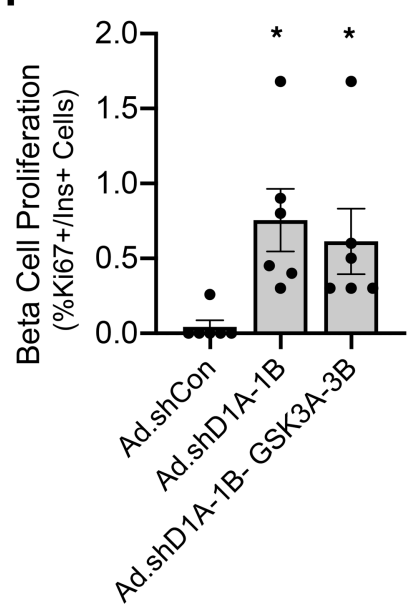

Figure 3. Silencing GSK3 family members alone or in combination does not induce human $\boldsymbol{\beta}$ cell proliferation. (A) qPCR studies illustrating that silencing GSK $3 \alpha$ or GSK3 $\beta$ attenuates expression of the corresponding gene. Data are shown as mean \pm SEM of 5 human islet donors. ${ }^{* * *} P<0.001$ vs. the Ad.shCon control by paired 2-tailed $t$ test. (B) Immunoblots of human islets treated with adenoviruses silencing GSK3 $\alpha$ or GSK3 $\beta$, demonstrating effective reductions in the corresponding proteins. (C) Effect on human $\beta$ cell proliferation of silencing CSK3 $\alpha$ or GSH3 $\beta$ compared with silencing DYRK1A. Data are shown as mean \pm SEM of 4 human islet donors. ${ }^{* * *} P<0.001$ vs. the Ad.shCon control and ${ }^{\# \#} P<0.001$ vs. Ad. shD1A, all by 1-way ANOVA with Bonferroni's multiple-comparisons test. (D) Illustration of an adenovirus designed to silence DYRK1A, DYRK1B, GSK3 $\alpha$, and GSH3 $\beta$ simultaneously. (E) qPCR demonstrating that the single virus in $\mathbf{D}$ silences its cognate targets. Data are shown as mean $\pm \mathrm{SEM}$ of 4 human islet donors. ${ }^{*} P<0.05$; ${ }^{* *} P<0.01$; ${ }^{* * *} P<0.001$ vs. the Ad.shCon control by paired 2-tailed $t$ test. (F) The effect on human $\beta$ cell proliferation of silencing DYRK1A and DYRK1B vs. both DYRKs and both GSKs. Ad.shCon is a virus expressing a control shRNA sequence directed against $\beta$-galactosidase. Data are shown as mean \pm SEM of 6 human islet donors. ${ }^{*} P<$ 0.05 vs. Ad.shCon by 1-way ANOVA with Bonferroni's multiple-comparisons test.

TG003 induced comparable maximal degrees of proliferation as harmine, INDY, 5-IT, and GNF4877 but with a right-shifted dose-response curve, yielding an $\mathrm{EC}_{50}$ of $\sim 20 \mu \mathrm{M}$. CC-401 displayed lower maximal proliferative efficacy but a comparable $\mathrm{EC}_{50}$ as compared with harmine. Together, these findings indicate that 5-IT and GNF4877 are the most potent human $\beta$ cell mitogenic compounds among the 8 studied. Note that most compounds also inhibited proliferation at the higher doses.

Effects of DYRK1A, DYRK1B, and GSK3ß overexpression on 5-IT-and GNF4877-induced proliferation. Since 5-IT and GNF4877 appear to be more potent drivers of human $\beta$ cell proliferation than harmine, we wondered whether they might exert this superior efficacy via targets other than, or in addition to, DYRK1A and DYRK1B. To explore this possibility, we adenovirally overexpressed DYRK1A and DYRK1B in human islets treated with the 3 DYRK1A inhibitors (Supplemental Figure 1), reasoning that if the mitogenic effects of 5-IT and GNF4877 were mediated exclusively via interfering with DYRK1A or DYRK1B, then providing an excess of DYRK1A and DYRK1B should effectively block their mitogenic ability. As expected, and as reported by ourselves and others $(5,7,8)$, overexpressing DYRK1A at high levels almost completely blocked the ability of harmine to induce $\beta$ cell proliferation (Figure 6A). Perhaps, predicted by Figure 2, B and G, overexpression of DYRK1B also blocked 

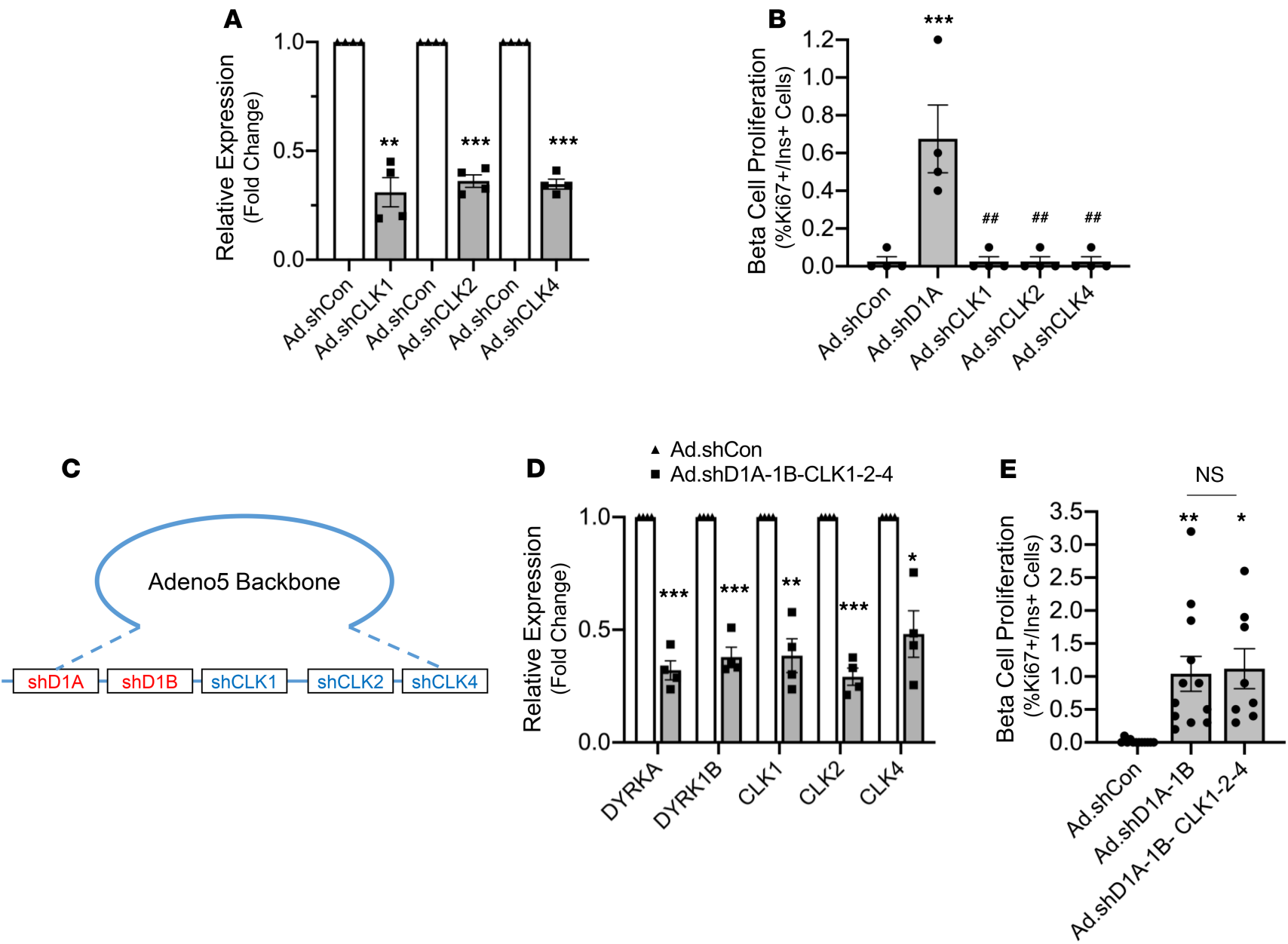

Figure 4. Silencing CLK family members alone or in combination does not induce human $\boldsymbol{\beta}$ cell proliferation. (A-E) Displayed as in Figure 3. Silencing CLKs 1,2 , and/or 4 can be accomplished alone or in combination, but silencing them in human islets has no effect on proliferation, whereas silencing DYRK1A alone or in combination with DYRK1B is effective. Data are presented as \pm SEM, and numbers of human islet donors studied in each panel are: (A) 4; (B) 4; (D) 4; and (E) 8-10 as shown. (A and D) ${ }^{*} P<0.05$; ${ }^{* *} P<0.01$; ${ }^{* *} P<0.001$ vs. the Ad. shCon control by paired 2-tailed $t$ test. (B and E) ${ }^{*} P<0.05$; ${ }^{* *} P<0.001$ vs. the Ad.shCon control; ${ }^{\#} P<0.001$ vs. Ad.shD1A. Comparisons with $P>0.05$ are labeled NS by 1-way ANOVA with Bonferroni's multiple-comparisons test.

the proliferative effects of harmine - and to the same degree as overexpression of DYRK1A (Figure 6A). DYRK1A and DYRK1B overexpression both also effectively blocked proliferation in response to 5-IT, comparable with results observed with harmine (Figure 6A), suggesting that the effects of 5-IT are largely or entirely mediated by inhibition of DYRK1A and DYRK1B. In contrast, while DYRK1A and DYRK1B overexpression did attenuate the proliferative effect of GNF4877, it was only reduced by about $65 \%$, significantly less pronounced than with comparable and simultaneous experiments with harmine and 5-IT. These results are consistent with the possibility that the greater efficacy of GNF4877 reflects its interactions with additional targets.

GNF4877 has been reported to be a GSK3 $\alpha$ and GSK3 $\beta$ inhibitor in rat $\beta$ cells $(8,14,15)$. To explore the possibility that GSK3 inhibition may contribute to the efficacy of GNF4877 in human $\beta$ cells, we queried whether the mitogenic efficacy of harmine could be enhanced by addition of GSK3 $\alpha$ and GSK3 $\beta$ inhibitors. First, as predicted based on Figure 5A, a maximally effective dose of GNF4877 (3 $\mu \mathrm{M})$ yielded a slightly higher rate of proliferation than a maximally effective dose of harmine $(10 \mu \mathrm{M})$ (Figure 6B). As predicted based on Figure 1, two different GSK3 $\alpha$ and GSK3 $\beta$ inhibitors, tideglusib and CHIR-99021, had no effect on human $\beta$ cell proliferation on their own. However, when the 2 GSK3 $\alpha$ and GSK3 $\beta$ inhibitors were added to harmine, they significantly accentuated proliferation, mimicking the proliferation observed with GNF4877. These findings are compatible with the possibility that the superior efficacy of GNF4877 may reflect its ability to inhibit both DYRK1A and DYRKB, as well as GSK3 $\alpha$ and/or GSK3 $\beta$. To explore this possibility further, we silenced GSK3 $\alpha$ or GSK3 $\beta$ in human islets treated with harmine, 5-IT, and 

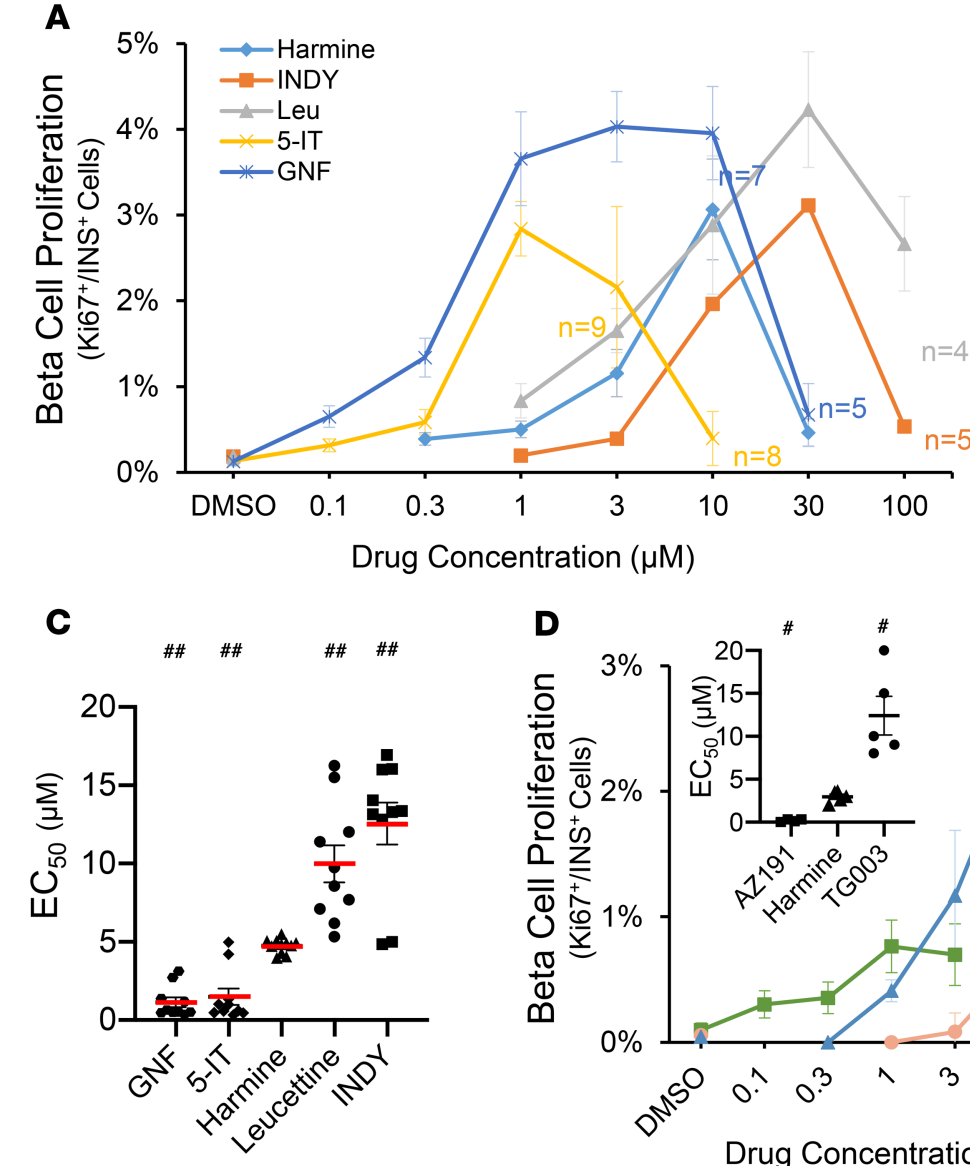
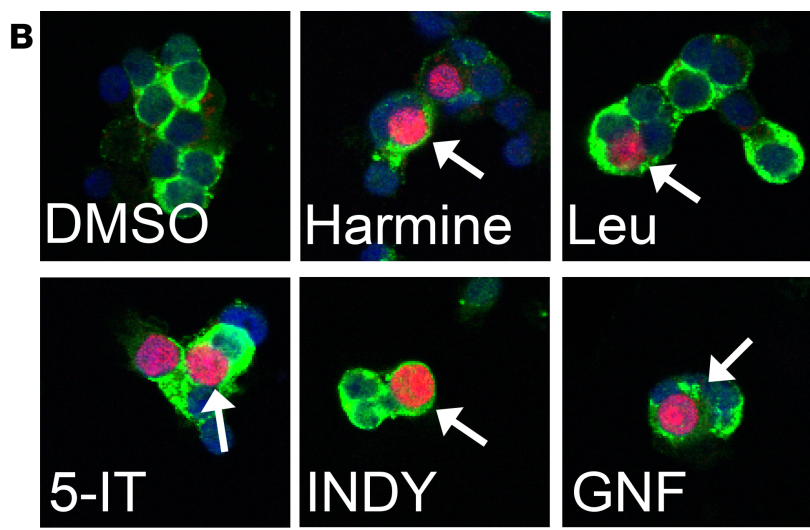

Insulin Ki67
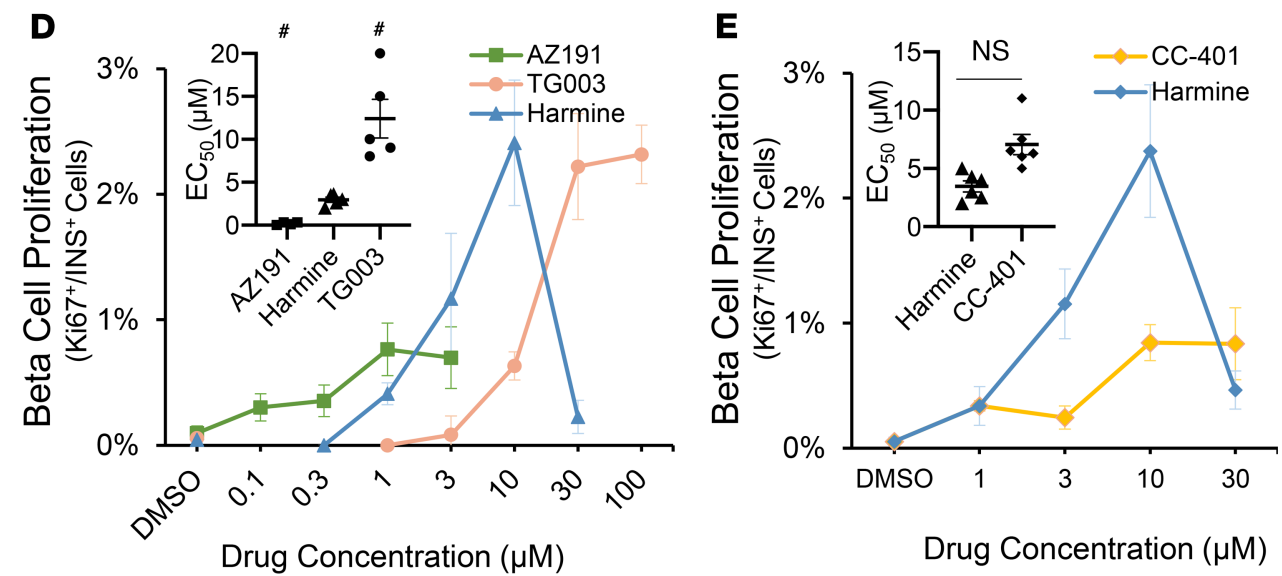

Figure 5. Complete dose-response curves for putative DYRI1A inhibitor drugs. (A) Dose-responses for harmine, INDY, leucettine-41, 5-IT, and GNF4877. All drugs were tested in the same 10 human islet preparations, except where lower $n$ is indicated, reflecting lack of human islet availability. Data are shown as mean \pm SEM. Note that each compound induces human $\beta$ cell proliferation, and that harmine, INDY, and leucettine-41 are similar in potency. Also note that the curves for 5-IT and GNF4877 are shifted to the left, indicating that they are approximately 10 -fold more potent mitogens than the other 3 . Finally, note that each drug loses efficacy at concentrations higher than its maximally effective dose. (B) Examples of Ki67-insulin coimmunolabeling for each drug. The faint blue in the background of some panels represents blue DAPI nuclear staining. Original magnification, $40 \times$. (C) Individual EC ${ }_{50}$ values for each compound in each human islet preparation. Each symbol represents a different human islet donor $\mathrm{EC}_{50}{ }^{*} \mathrm{\#} P<_{0.001}$ as compared with harmine by 1-way ANOVA, Bonferroni's multiple-comparisons test. (D) Dose-response curves for 2 additional putative DYRK1A inhibitors, AZ191 and TC003, com-

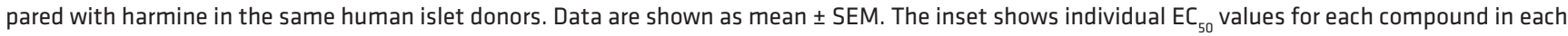
human islet preparation. Each symbol represents a different human islet donor $\mathrm{EC}_{50}{ }^{*} P<0.05$ as compared with harmine by 1 -way ANOVA, Bonferroni's multiple-comparisons test. The numbers of human islet donors studied were 4 for AZ191 and 6 for both harmine and TG003. Note that each is inferior to harmine, and by extension to A, to the rest of the DYRK1A inhibitor class. (E) Dose-response curve for CC-401, compared with harmine in the same human islet donors. Data are shown as mean \pm SEM. Inset shows individual $\mathrm{EC}_{50}$ values for each compound in each human islet preparation. Each symbol represents a different human islet donor $\mathrm{EC}_{50}$. Comparisons with $P>0.05$ are labeled NS by 1-way ANOVA, Bonferroni's multiple-comparisons test. Six human islet donors were studied for both harmine and CC-401.

GNF4877. As observed in Figure 3F, silencing GSK3 $\alpha$ or GSK3 $\beta$ in human islets had little effect and also had no additional effect on human islets treated with harmine, 5-IT, or GNF4877 (Figure 6C). To further explore a possible role for GSK3 $\beta$ in human $\beta$ cell proliferation, we asked whether overexpression of a constitutively active isoform of GSK3 3 (28) in human islets might attenuate the mitogenic efficacy of harmine, GNF, or 5-IT. As can be seen in Figure 6D, overexpression of constitutively active GSK3 $\beta$ in human islets had no effect on the mitogenic effects of harmine, 5-IT, or GNF4877. Collectively, within the well-documented variability among human islet preparations $(5-13,29)$ and the limited ability to study every drug at every possible dose in every human islet preparation, the findings in Figure 6 are compatible with the possibility that GSK3 $\alpha$ or GSK3 $\beta$ inhibition by GNF4877 may provide a modest contribution to the superior mitogenic potency of GNF4877 as compared with harmine. On the other hand, if this is true, this modest contribution is unlikely to explain the 10-fold-greater mitogenic potency of GNF4877 and 5-IT on human $\beta$ cells observed in Figure 5, A and C. 

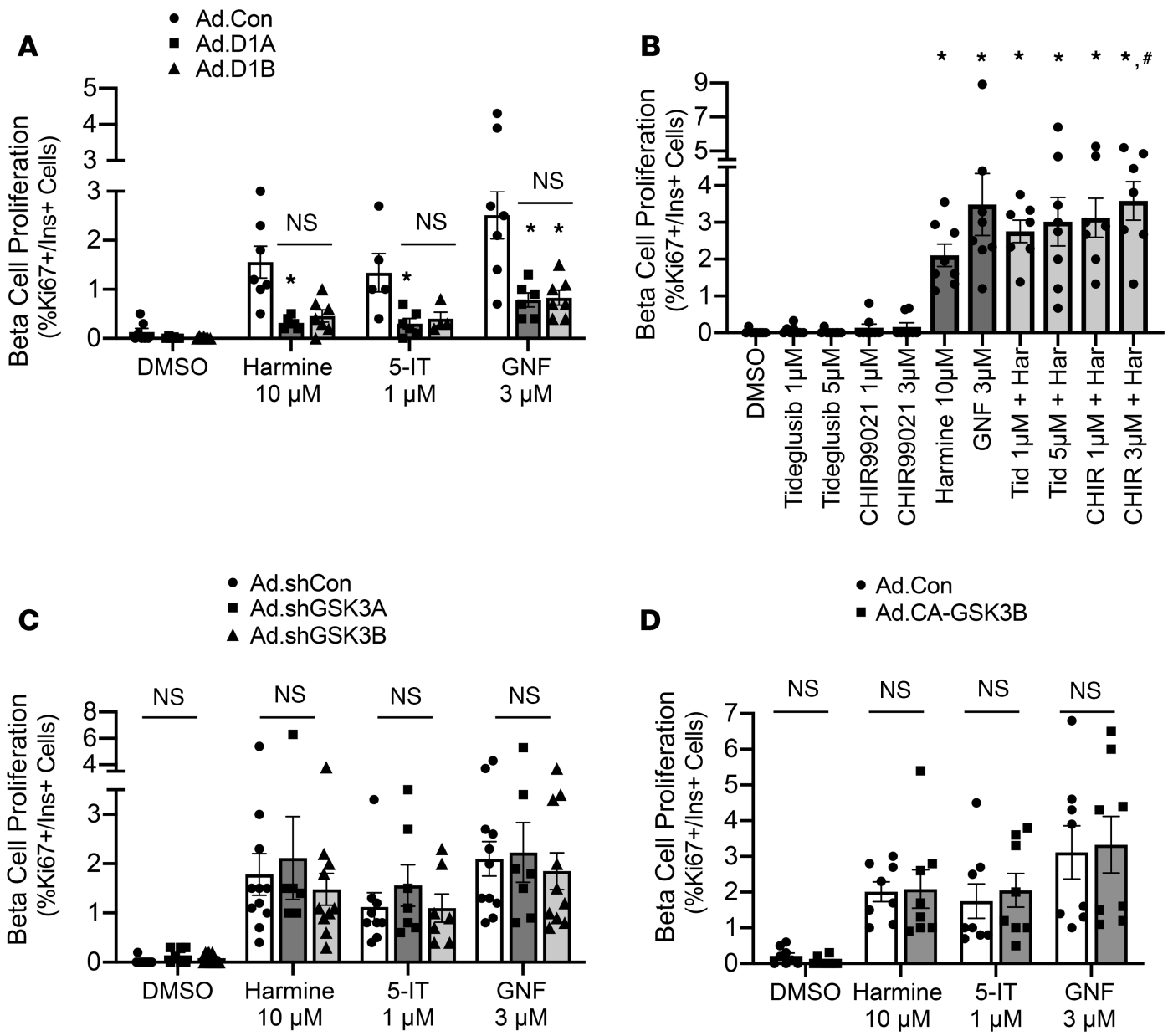

Figure 6. Exploring the role of DYRK1A, DYRK1B, GSK3 $\alpha$, and GSK3 $\beta$ in harmine-, 5-IT-, and GNF4877-induced proliferation. (A) Adenoviral overexpression of DYRK1A or DYRK1B human $\beta$ cell proliferation induced by harmine, 5-IT, or GNF4877 in the doses shown. Note that overexpression of either DYRK1A or DYRK1B markedly attenuates the induction of proliferation induced by harmine or 5-IT and, to a lesser extent, GNF4877. (B) The effect of the GSK3 $\alpha$ and GSK3 $\beta$ inhibitors, tideglusib and CHIR99021, on human $\beta$ cell proliferation alone or in combination with harmine. GNF4877 and harmine serve as positive controls, and GNF4877 generates a larger effect than harmine, as in Figure $5 .{ }^{*} P<0.05$ vs. DMSO control, ${ }^{*} P<0.05$ vs. harmine alone, all by 1-way ANOVA with Bonferroni's multiple-comparisons test. Note that the addition of tideglusib or CHIR99021 to harmine seems to enhance its effects, rendering it comparable to GNF4877. (C) The effect of silencing GSK3 $\alpha$ or GSK3 $\beta$ on proliferation induced by harmine, 5-IT, or GNF4877. Note that, in this paradigm, silencing the GSKs appears to have little effect on human $\beta$ cell proliferation. NS, $P>0.05$ by paired 2 -tailed $t$ test. (D) The effect of expressing a constitutively active GSK3 $\beta$ on harmine-, 5-IT-, and GNF4877-induced proliferation. In this paradigm, expressing a constitutively active GSK3 $\beta$ isoform has no effect on the mitogenic efficacy of harmine, 5 -IT, or GNF4877. Ns, $P>0.05$ by paired 2-tailed $t$ tests. Data are shown as mean $\pm \mathrm{SEM}$, and the numbers of human islet donors in each panel are indicated by the symbols. Collectively, the studies in this panel, combined with those in B and C and Figure 3, suggest that if GSK3 inhibition contributes to the enhanced efficacy to GNF4877 or 5-IT as compared with harmine, it is a small contribution. Data are shown as mean $\pm \mathrm{SEM}$. In $\mathbf{A}, \mathbf{C}$, and $\mathbf{D},{ }^{*} P<0.05$ vs. Ad.Con by 2 -way ANOVA with Bonferroni's multiple-comparisons test. NS, $P>0.05$ all by 2-way ANOVA with Bonferroni's multiple-comparisons test.

Comparison of kinome scans for 5-IT and GNF4877 with that of harmine. Since 5-IT and GNF4877 appear to be more potent than harmine in driving human $\beta$ cell proliferation (Figure 5A), and since a candidate approach to identify additional targets for 5-IT and GNF had been unfruitful, we next employed an unbiased approach, performing kinome scans for 5-IT and GNF4877 against the 468 kinases in the DiscoverX panel, all at $10 \mu \mathrm{M}$ (Figure 7 and Supplemental Table 1), exactly as we had for harmine (Supplemental Table 1); we hoped to find a small subset of targets that might explain the greater efficacy of GNF4877 and 5-IT as compared with harmine, as well as leucettine-41 and INDY, for which kinome scans are also available $(9,10)$. To our surprise, as compared with harmine at the same concentration, GNF4877 and 5-IT displayed a far greater number of potential targets (selectivity scores of 103 for GNF4877, 39 for 5-IT, and 2 for harmine), making pursuit of their additional mechanisms of action and additional targets untenable in practical terms. As reported previously $(7,8)$, and as expected, lower doses of 5-IT and GNF4877 are more selective than higher doses. 
A

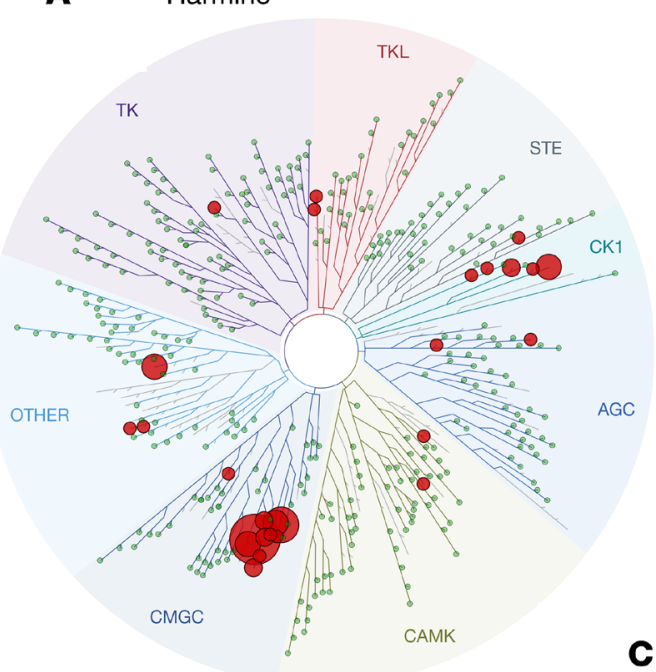

C GNF4877
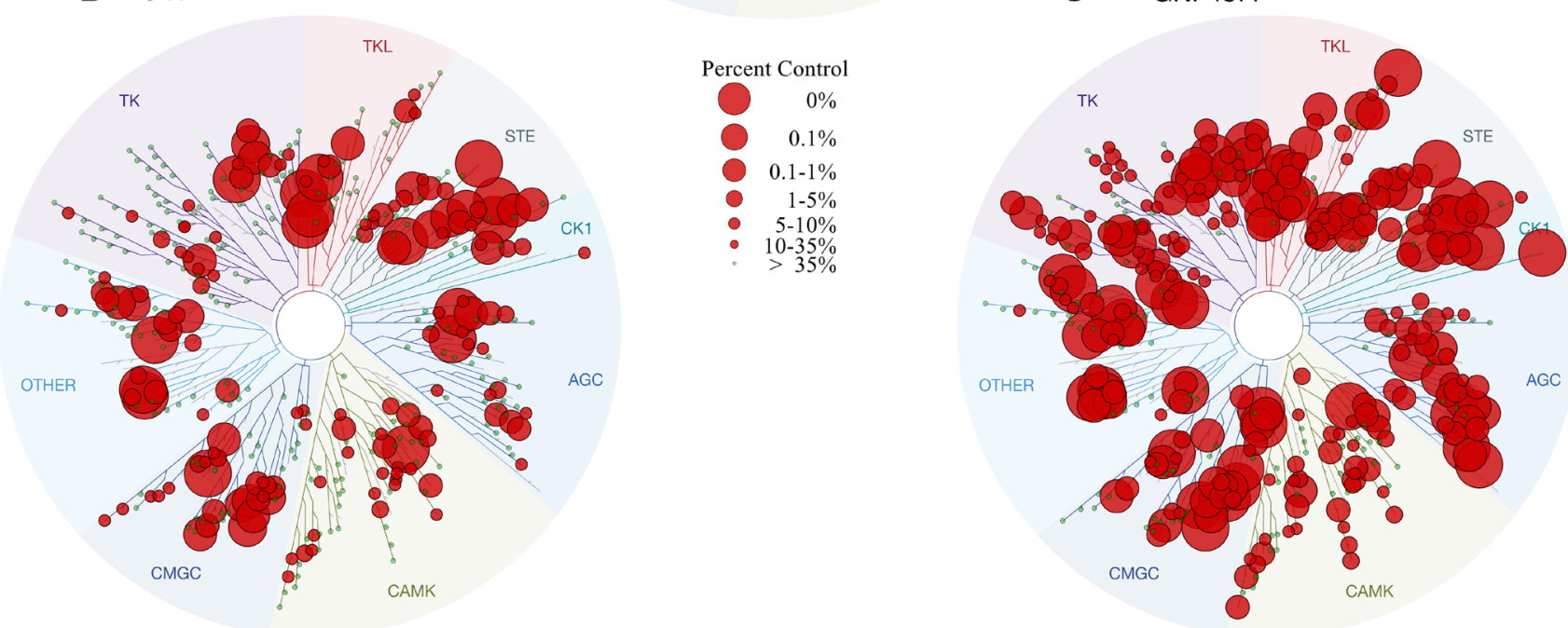

Figure 7. Kinome screens for harmine, 5-IT, and GNF4877. All compounds were screened in the same DiscoverX kinome screen assay at the same $10-\mu \mathrm{M}$ dose. Complete lists of "hits" for harmine, 5-IT, and GNF4877 are shown in Supplemental Table 1. The important point is that both 5-IT and GNF4877 hit many additional kinases that may enhance human $\beta$ cell proliferation but also provide challenges in drug development and enhancing selectivity.

\section{Discussion}

We report a number of important observations. First, we provide the first comprehensive comparative documentation to our knowledge defining the relative potency among the small molecule DYRK1A inhibitor family as agonists for human $\beta$ cell proliferation. Second, we employ a comprehensive comparison of small molecule pharmacologic inhibitors, combined with silencing of putative specific target genes, and comprehensive kinome scans to define the principal mitogenic targets of the DYRK1A inhibitor class. Third, we demonstrate that there is an unanticipated compensatory increase in DYRK1B abundance in human islets when DYRK1A is silenced. Fourth, we observe that silencing DYRK1B contributes to the proliferative efficacy of DYRK inhibitors. Fifth, we show that CLK family of CMGC members, identified in kinome scans as candidate regulators of human $\beta$ cell proliferation, do not contribute to proliferation driven by the DYRK1A inhibitor class. Seventh, similarly, we find that GSK3 $\alpha$ and GSK3 $\beta$ are not likely targets for proliferation-inducing drugs of the DYRK1A inhibitor class, with the possible exception of GNF4877, where a small degree of augmentation appears possible. Eighth, and importantly, in the course of performing these studies, we developed a panel of adenovirus tools able to deploy multiple shRNAs in a single adenovirus; this is especially useful for studies in human $\beta$ cell research and likely well beyond. 
Kinome scans have been reported previously for harmine, leucettine-41, INDY, 5-IT, GNF4877, and CC-401 (7-13). These scans were performed in different laboratories and companies, exploring 253-468 different kinases, tested at a range of doses, from $100 \mathrm{nM}-10 \mu \mathrm{M}$. In each case, the CMGC class was identified as the lead class, and in each case, DYRK1A was identified as a, or the, lead hit. On the other hand, potentially relevant inhibition in most cases has been observed for other DYRKs, CLKs, and GSK3s (7-13). In some screens, the casein kinases CSNK1D and CSNK1E also have been identified. The current study - through RNA-seq profiles, kinome screens, pharmacologic studies, and genetic silencing approaches - makes it clear that the DYRKs 2, 3, and 4; the CLKs; and GSK3s are unlikely to be involved in the human $\beta$ cell proliferative response to DYRK1A inhibitors, with the possible exception of GNF4877, as discussed below.

One surprise was the observation that DYRK1B increased when DYRK1A was silenced (Figure 2, $A$ and B) and that simultaneous silencing of DYRK1A and DYRK1B led to higher rates of human $\beta$ cell proliferation than silencing DYRK1A or DYRK1B individually (Figure 2G). These observations, together with the kinome scans herein (Figure 7 and Supplemental Table 1) and elsewhere (7-13) showing that the DYRK1A inhibitors effectively inhibit DYRK1B in addition to DYRK1A, and the observation that DYRK1B is readily detectable in $\beta$ cells (Table 1 and refs. 23-25), support the unanticipated conclusion that optimal mitogenic efficacy of the DYRK1A inhibitor class requires combined inhibition of both DYRK1A and DYRK1B. While it is known the NFaT class of transcription factors are downstream mediators of the actions of DYRK1A inhibition (5, 12, 13, 17, 19), how DYRK1B inhibition might fit into this paradigm or other mechanisms is unknown and requires additional study. Similarly, why DYRK1B abundance increases when DYRK1A is silenced in human islets (Figure 2, A and B) is unknown, although it does not appear to be a result of increased DYRK1B mRNA abundance (Figure 2C). The apparent compensatory increase in DYRK1B protein abundance should be the focus of additional studies. Most importantly, these findings make the unexpected and important point that an optimal human $\beta$ cell mitogenic drug must be a potent inhibitor of both DYRK1A and DYRK1B.

While the DYRK, CLK, and GSK3 families appear most often in DYRK1A inhibitor kinome scans (7-13), other kinases have been observed, including CSNKs noted above, haspin, and homeodomain interacting protein kinases (HIPKs) 2 and 3, suggesting that inhibition of these kinases may be relevant to human $\beta$ cell proliferation. It was not possible to prepare adenoviruses to silence each member of each of the CLK, GSK3, haspin, CSNK, and HIPK families in every possible combination, all in human $\beta$ cells, with and without and simultaneous silencing of DYRK1A and DYRK1B; however, it seems unlikely that these are relevant targets. More specifically, the CSNKs seem unlikely participants because several mitogenically effective DYRK1A inhibitors are not CSNK inhibitors. For example, a modified potent harmine analog and DYRK1A inhibitor, compound 2-2 is not a CSNK inhibitor $(12,13)$, nor are 5-IT (7) and leucettine-41 (9). Similarly, HPK2 and HPK3 are not inhibited by leucettine-41, GNF4877 (8), or 5-IT (7) at effective doses. On the other hand, haspin is inhibited by harmine, 5-IT, and, to a lesser extent, GNF4877 (Supplemental Table 1 and refs. 7, 12,13) and remains a viable candidate for a role in driving human $\beta$ cell proliferation in response to DYRK1A inhibitors. This merits future study. Collectively, the available kinome screen data support the conclusion that the principal $\beta$ cell proliferation-relevant kinase targets of harmine, INDY, leucettine, GNF4877, and 5-IT are DYRK1A and DYRK1B.

Of course, it is not essential that relevant targets of DYRK1A inhibitors must be protein kinases; scaffold proteins, signaling proteins, receptors and signaling molecules, other cellular proteins, and lipid kinases may constitute additional relevant targets. For example, as noted above, harmine treatment of human islets reduces abundance of SMAD proteins that signal downstream of the TGF- $\beta$ superfamily of receptors (6) and reduces abundance of the cell cycle inhibitor p27 $7^{\mathrm{KIP} 1}$ in rat islets (11). The Alzheimer-associated protein Tau and the cell cycle regulators Lin-52 and D-cyclins are also known to be regulated by DYRK1A inhibitors $(11,20,21)$, but whether these are relevant to their induction of human $\beta$ cell replication is unknown. Thus, while it is clear that, among the protein kinase family, DYRK1A and DYRK1B are the relevant targets of the DYRK1A inhibitor class, it is possible - indeed likely — that additional targets of relevance exist. Identifying and validating these presumptive additional targets will require extensive advanced proteomic, metabolomic, and lipidomic studies.

DYRK1A inhibitors such as harmine, INDY, leucettine, 5-IT, and GNF4877 are effective at certain doses but inhibit proliferation at higher doses, as summarized in Figure 5. While the underlying mechanisms responsible for this phenomenon are uncertain, a likely explanation is that high doses of a given DYRK1A inhibitor also inhibit kinases essential for cell cycle activation or progression. As a potential 
example, several (but not all) DYRK1A inhibitors inhibit the cell cycle-activating kinase CDK7 (Figure 7, Supplemental Table 1, and refs. 7, 8, 11-13). CDK7 is an essential member of the CDK-activating kinase (CAK) complex that activates the CDK family, which phosphorylates the $\mathrm{pRb}$ (retinoblastoma protein) family as an essential first step to cell cycle entry. CDK7 is, thus, an example of an off-target antikinase that may reduce the efficacy of the DYRK1A inhibitor family. From a drug-development viewpoint, this type of information is helpful in the design of future DYRK1A/DYRK1B inhibitors.

A final important outcome of these studies is a technical one. Since adding multiple adenoviruses (or other types of viruses) to human islets (or presumably most cell types) with the resultant higher multiplicity of infection (MOI) and higher total virus dose is toxic, we built adenoviruses capable of delivering multiple shRNAs in a single virus. This provides 2 advantages over conventional adenoviral gene deliver approaches. First, it permits silencing of multiple targets, without administering toxic doses of adenovirus. Second, in contrast to a panel of individual shRNA-bearing adenoviruses, it ensures that all of the shRNA cargoes enter a given single cell. Thus, in conventional approaches, 1 Ad.shRNA virus silencing DYRK1A might enter 1 cell, and a second virus silencing DYRK1B might enter another and reveal no addition effects on proliferation. The multi-shRNA adenovirus system permits simultaneous delivery of up to 7 different shRNAs to a single cell.

This study has limitations. First, we used Ki67-insulin coimmunolabeling as a surrogate for $\beta$ cell proliferation. We believe this is justified because it allows rapid sample screening and is not associated with activation of cell death pathways in human islets treated with DYRK1A inhibitors $(5,6)$ and because we have shown that it correlates nicely with increases in actual human $\beta$ cell numbers (6). Second, while we effectively silence the CLK family at the mRNA level, we do not demonstrate effective reduction in CLK proteins in Figure 4. This reflects a lack of antisera that can assess and distinguish among CLK family members. Third, as emphasized above, it is likely that the DYRK1A inhibitors influence additional nonkinase targets not identified herein. Identifying these additional targets will require complex proteomic, metabolomic, and lipidomic approaches, such as pull-down experiments with DYRK1A inhibitors and appropriate controls. Fourth, in the case of GNF4877, while we conclude that the principal mitogenic effects on human $\beta$ cells are mediated by DYRK1A and DYRK1B inhibition, we are unable to definitively identify the kinases or other target molecules responsible for the remaining 30\% of the proliferation implied in Figure 6 . As shown in Figure 7 and Supplemental Table 1, GNF4877 has a particularly broad and complex range of kinase targets - and likely a similarly broad range of additional nonkinase targets. Optimizing the selectivity of next-generation GNF4877 analogs will be necessary for translation to human clinical use. Fifth, we did not assess measures of cell death in this study of human $\beta$ cell regeneration because prior studies have found no evidence that DYRK1A inhibitors induce $\beta$ cell death at maximally effective mitogenic doses or in vitro or in vivo (5-8). Sixth, as described by ourselves and others, DYRK1A inhibitors are not specific for human $\beta$ cells $(5-8,16)$; they induce proliferation in $\alpha$ cells, $\delta$ cells, and other islet cell types. This lack of pure $\beta$ cell specificity underlies the recent emphasis by diabetes research funding agencies for the discovery and validation of molecules that are able to target therapeutic molecules exclusively and effectively to the human $\beta$ cell. Seventh, while we and other authors suggest that DYRK1A is inhibited in human $\beta$ cells, no group to our knowledge has ever actually measured DYRK1A kinase activity in a pure population of human $\beta$ cells for technical reasons. Finally, these studies were performed in dispersed human islets in the absence of their normal, in vivo, physiologic cues and context, and in the absence of in vivo pharmacokinetic and tissue distribution considerations. In vivo efficacy and selectivity will require extensive in vivo studies with future lead compounds.

In summary, the human $\beta$ cell regenerative biology field now has a number of small molecule drugs that effectively increase $\beta$ cell proliferation. The next challenges will be enhancing the selectivity and potency of DYRK1A/DYRK1B inhibitors through structure-based drug design (11-13), combining them with additional synergizing molecules - exemplified by the addition of TGF- $\beta$ superfamily inhibitors (6) - and developing reagents and tools to deliver potent DYRK1A inhibitors to the $\beta$ cell in vivo in a targeted manner, avoiding other tissues and cell types that express DYRK1A and DYRK1B.

\section{Methods}

Human pancreatic islets. HIPPA-compliant deidentified islets from adult cadaveric pancreas donors were obtained from the NIH/NIDDK-supported Integrated Islet Distribution Program (IIDP) (http://iidp.coh. org), from Prodo Laboratories, or from Patrick MacDonald at the Alberta Diabetes Institute (Edmonton, Alberta, Canada), as summarized in Supplemental Table 2. A total of 54 human islet preparations were used. Donors ranged in age from 18-69 years (mean \pm SEM, $44.3 \pm 13.5$ ); 17 were female and 37 were male. 
Mean BMI was $30.2 \pm 6.8$ (range 18.2-42.8), and cold ischemia time was 589 minutes (range 121-1338 minutes). Purity ranged from $75 \%-95 \%$ (mean $86.3 \pm 6.0$ ).

Chemicals. Drugs sources were as follows: INDY (Tocris Biosciences, 4997), BrdU substrate (GE Healthcare, RPN20), Harmine (MilliporeSigma, 286044), leucettine-41 (Adipogen, AG-MR-C0023-M005), 5-IT (MilliporeSigma, I100), GNF4877 (Xcessbio, M60294), AZ191 (Selleckchem, S7338), TG003 (Cayman Chemical, 10398), CC-401(Cayman Chemical, 18867), CHIR99021 (Cayman Chemical, 13122), tideglusib (Selleckchem, S2823), PF-4800567 (Cayman Chemical, 19171), and PF-670462 (Cayman Chemical, 14588).

Adenoviruses and transduction. Adenoviruses expressing shRNAs directed against DYRK1A and LacZ have been described previously (5). Adenoviruses expressing shRNAs directed against other DYRKs, GSK3s, and CLKs were prepared in the same manner. shRNA target sequences are shown in the following paragraph. The adenovirus overexpressing human DYRK1A has been reported previously (5). The adenoviruses overexpressing human DYRK1B were prepared using cDNA encoding DYRK1B obtained from Harvard PlasmID Database (http://plasmid.med.harvard.edu). The adenovirus expressing constitutively active GSK3 $\beta$ was prepared using cDNA encoding GSK3 $\beta$ S9A from Addgene (http://www.addgene.org). Adenoviruses were used at an MOI of 150 for 2 hours, and cells were harvested for study 96 hours later.

Generation of single adenoviruses expressing shRNAs against multiple target genes. In order to silence multiple genes in 1 virus, we first made constructs that silence individual genes of interest using U6 RNAi entry vector (Invitrogen, 45-0511). We then cloned the U6-shRNA sequence by PCR and ligated it into modified PENTR 2B Gateway vector (Thermo Fisher Scientific, A10463) as per the manufacturer's instructions. More specifically, to make the Ad.shRNA DYRK1A-DYRK1B (Ad.shD1A-1B), a U6-DYRK1A-short hairpin (U6-DYRK1A-sh) cassette was first cloned by PCR and ligated into PENTR 2B; then, a U6-DYRK1B sh cassette was cloned and ligated into the construct previously inserted into the U6-DYRK1A sh cassette. To make Ad.shRNA shD1A-1B-D2, a U6-DYRK2 sh cassette was cloned and ligated with the construct that contained the U6-DYRK1A-DYRK1B sh cassette. Using this method, we prepared a variety of constructs, including Ad.shD1A-1B, Ad.shD1A-1B-D2, Ad.shD1A-1B-D2-D3, Ad.shD1A-1B-D2-D3-D4, Ad.shD1A-D1B-GSK3A-GSK3B, and Ad.shD1A-1B-CLK1-CLK2-CLK4 (Ad.shD1A-1B-CLK1-2-4). Then, we used the Gateway recombination system described above to recombine the U6-shRNA cassettes into the adenovirus backbone, producing adenovirus as described previously (5). For shRNA target sequence, Ad.shDYRK1A against DYRK1A has been reported previously (5), DYRK1B: GCTGGAGCGCTACGAAATTGA, DYRK2: GCTCACGACACAACCAAATGG, DYRK3: GGTAGAACAGCTGTTTCAAGA, DYRK4: GGATTCATCAGTCTCGGAACC, GSK3ß: GGTATATCAAGCCAAACTTTG, GSK3 $\alpha$ : GGGAACTAGTCGCCATCAAGA, CLK1: GCATAGGATGCATTCTTATTG, CLK2: GGGCCTTAGCACCTTCGATTT, CLK4: GCTTTCTGCCATTTCAAATTG.

Human islet dispersion. Islets were centrifuged at $380 \mathrm{~g}$ for 10 minutes, washed twice in PBS, resuspended in $1 \mathrm{~mL}$ of Accutase (MT25058CI, Thermo Fisher Scientific), and incubated for 10 minutes at $37^{\circ} \mathrm{C}$. During this digestion, the islets were dispersed by gentle pipetting up and down every 5 minutes for 10 seconds. Complete RPMI medium containing $11 \mathrm{mmol} / \mathrm{L}$ glucose and $1 \%$ penicillin/streptomycin (15140122, Thermo Fisher Scientific) with 10\% FBS (F2442, MilliporeSigma) was then added to stop the digestion. The cells were then centrifuged for 5 minutes at $380 \mathrm{~g}$, the supernatants were removed, the pellet was resuspended in complete medium, and cells were then plated on coverslips with $30 \mu \mathrm{L}$ cell suspension per coverslip. Poly-D-Lysine/laminin-treated (BD354688, VWR Scientific) cover slides or chamber slides were used. Cells were then allowed to attach for 2 hours at $37^{\circ} \mathrm{C}$ or were transduced with adenovirus for 2 hours. After 2 hours, $500 \mu \mathrm{L}$ complete RPMI was added in each well to terminate the adenoviral transduction. Cells were cultured for 24-96 hours as described in the appropriate figure legends.

Compound treatments. For compound treatment, after dispersed islet cells were allowed to recover from dispersal on the coverslip for 24 hours, complete medium was replaced with the medium containing compounds for 96 hours, at the doses described in the figures.

$q P C R$ gene expression analysis. RNA was isolated and $\mathrm{qPCR}$ was performed as described previously $(5,6,20)$. Briefly, total human islet RNA was extracted using the RNeasy Micro Kit (Qiagen, 74004). qPCR was performed using SuperScript IV VILO Master Mix (Invitrogen, 11756500). Gene expression in dispersed islets was analyzed by real-time PCR performed using iTag Universe SYBR Green Supermix (Bio-Rad, 172-5124) on an ABI 7500 System. CYPA was used as the reference gene to normalize the gene expression. The experimental Ct was calculated using the algorithm enhancements provided by the equipment. Primers used are shown in Table 2. 
Table 2. PCR Primers Used For Gene Expression Analysis

\begin{tabular}{|c|c|}
\hline GSK $3 \alpha$ forward & CACAGTCGTAGCCACTCTAGG \\
\hline GSK $3 \alpha$ reverse & GTCCAGCTTACGCATGATCTG \\
\hline GSK $3 \beta$ forward & TGGTCGCCATCAAGAAAGTATTG \\
\hline GSK $3 \beta$ reverse & GCGTCTCTTTGGCTCGACTAT \\
\hline CLK1 forward & AGAGACCATGAAAGCCGGTAT \\
\hline CLK1 reverse & CATGTGAACGACGATGTGAAGT \\
\hline CLK2 forward & AAGCAGTTATGATGATCGTTCGT \\
\hline CLK2 reverse & GGCTATAATCGTTGCGTCTCTAG \\
\hline CLK4 forward & ATCGGGACCGGAGATACGTT \\
\hline CLK4 reverse & ATTCGATACCCGCTTTCAATGT \\
\hline CYPA forward & CACCGTGTTCTTCGACATTG \\
\hline CYPA reverse & TCAACTCACСACССTGACAC \\
\hline DYRK1A forward & GGATCGTTACGAAATTGACTCCT \\
\hline DRYK1A reverse & ACATAAACTCGCGTTTCAAATGC \\
\hline DYRK1B forward & ATGAACCAGCATGACACGGAG \\
\hline DYRK1B reverse & СTTGAGGTCGСAGTGAATGAT \\
\hline DYRK2 forward & CAGTGCTCACGACACAACCA \\
\hline DYRK2 reverse & ССGTCTATGAАTССTGTCСAG \\
\hline DYRK3 forward & ACAAACTTCGACAGTACGTGG \\
\hline DYRK3 reverse & САACTGGACGCTAAAACССТG \\
\hline DYRK4 forward & ATACACGTACATCCAAACCCG \\
\hline DYRK4 reverse & ССТGGAGGСТGTСTGAАTGAА \\
\hline
\end{tabular}

Immunocytochemistry. Immunocytochemistry was performed on 4\% paraformaldehyde-fixed (15 minutes), accutase-dispersed human islets plated on coverslips as described (5). Primary antisera were Ki67 (RM-9106-s1, Thermo Fisher Scientific) and insulin (A0564, DAKO). Species-specific secondary antisera (mouse Alexa Fluor 488 [A-11029, Invitrogen], rat Alexa Fluor 594 [A-11007, Invitrogen], rabbit Alexa Fluor 594 [A11037, Invitrogen], or guinea pig Alexa Fluor 488 [A-11073, Invitrogen]) were selected as appropriate. In each experiment, a minimum of $1000 \beta$ cells was counted. The numbers of Ki67-posiitve cells are expressed as a percent of total insulin-positive cells.

Immunoblots. Immunoblots were performed on whole human islets as described in detail previously (5, 6) using the following antisera: DYRK1A (MilliporeSigma, D1694), DYRK1B (Cell Signaling Technologies, 5672), DYRK2 (Abcam, ab37912), GSK3 $\alpha / 3 \beta$ (Cell Signaling Technologies, 5676), and GAPDH (Sc-25778, Santa Cruz Biotechnology Inc.).

Kinome screens. LeadHunter kinome screens for harmine, 5-IT, and GNF4877, all at $10 \mu \mathrm{M}$, were performed by DiscoverX Corp. against 468 kinases as detailed in Supplemental Table 1. Briefly, this is a competitive binding or displacement assay that assesses residual binding to a solid-phase-bound small molecule test ligand against 468 different recombinant kinases following the addition of a small molecule of interest — in this case, harmine, 5-IT, or GNF4877. Results are expressed as percent of residual binding as compared with reactions containing no competing ligand. Complete assay details are provided at http://www. discoverx.com/technologies-platforms/competitive-binding-technology/kinomescan-technology-platform. Selectivity scores are calculated and provided by DiscoverX, as described at https://www.discoverx. com/services/drug-discovery-development-services/kinase-profiling/kinomescan/scanmax.

Statistics. Data are presented as mean \pm SEM. One-way ANOVA with Bonferroni's multiple-comparisons test was used to determine differences between more than 2 groups, and paired 2-tailed Student's $t$ test was used to compare 2 groups, as described in the figure legends. $P$ values less than 0.05 were considered to be significant. Statistical analyses were performed using Microsoft Office Excel and GraphPad Prism statistical software.

Study approval. This study uses human cadaveric pancreatic islets purchased from the NIH/National Institute of Diabetes and Digestive and Kidney Diseases (NIDDK), City of Hope Medical Center, Prodo Laboratories, and The Alberta Diabetes Institute, who certify that organs are obtained with appropriate informed consent and institutional approval. All subjects are deidentified. 


\section{Author contributions}

CA, ES, KK, HL, SC, EK, and PW performed experiments. DKS, AGO, RS, and RJD served as consultants. AFS and PW conceived of the studies and wrote the manuscript.

\section{Acknowledgments}

The authors want to thank Bonnie and Joel Bergstein, and Lonnie and Tom Schwartz for their support of this work. We also thank the NIDDK-supported Einstein-Sinai Diabetes Research Center and its Human Islet and Adenovirus Core (HIAC), the NIDDK-supported Human Islet Research Network (HIRN), and Patrick MacDonald at the Alberta Diabetes Institute. This work was supported by the NIH grants R01 DK105015, R01 DK116904, R01 DK116873, UC4 DK104211, and P-30 DK 020541, as well as JDRF grant 2-SRA-2017 514-S-B.

Address correspondence to: Andrew F. Stewart, Diabetes Obesity Metabolism Institute, Atran 5, PO Box 1152, Icahn School of Medicine at Mount Sinai, One Gustave Levy Place, New York, New York 10029, USA. Phone: 212.241.3993; Email: andrew.stewart@mssm.edu.

1. Meier JJ, Bhushan A, Butler AE, Rizza RA, Butler PC. Sustained beta cell apoptosis in patients with long-standing type 1 diabetes: indirect evidence for islet regeneration? Diabetologia. 2005;48(11):2221-2228.

2. Campbell-Thompson M, et al. Insulitis and $\beta$-Cell Mass in the Natural History of Type 1 Diabetes. Diabetes. 2016;65(3):719-731.

3. Butler AE, Janson J, Bonner-Weir S, Ritzel R, Rizza RA, Butler PC. Beta-cell deficit and increased beta-cell apoptosis in humans with type 2 diabetes. Diabetes. 2003;52(1):102-110.

4. Cinti F, et al. Evidence of $\beta$-Cell Dedifferentiation in Human Type 2 Diabetes. J Clin Endocrinol Metab. 2016;101(3):1044-1054

5. Wang P, et al. A high-throughput chemical screen reveals that harmine-mediated inhibition of DYRK1A increases human pancreatic beta cell replication. Nat Med. 2015;21(4):383-388.

6. Wang P, et al. Combined Inhibition of DYRK1A, SMAD, and Trithorax Pathways Synergizes to Induce Robust Replication in Adult Human Beta Cells. Cell Metab. 2019;29(3):638-652.e5.

7. Dirice E, et al. Inhibition of DYRK1A Stimulates Human $\beta$-Cell Proliferation. Diabetes. 2016;65(6):1660-1671.

8. Shen W, et al. Inhibition of DYRK1A and GSK3B induces human $\beta$-cell proliferation. Nat Commun. 2015;6:8372.

9. Tahtouh T, et al. Selectivity, cocrystal structures, and neuroprotective properties of leucettines, a family of protein kinase inhibitors derived from the marine sponge alkaloid leucettamine B. J Med Chem. 2012;55(21):9312-9330.

10. Ogawa Y, et al. Development of a novel selective inhibitor of the Down syndrome-related kinase Dyrk1A. Nat Commun. 2010;1:86.

11. Abdolazimi Y, et al. CC-401 Promotes $\beta$-Cell Replication via Pleiotropic Consequences of DYRK1A/B Inhibition. Endocrinology. 2018;159(9):3143-3157.

12. Kumar K, Wang P, Sanchez R, Swartz EA, Stewart AF, DeVita RJ. Development of Kinase-Selective, Harmine-Based DYRK1A Inhibitors that Induce Pancreatic Human $\beta$-Cell Proliferation. J Med Chem. 2018;61(17):7687-7699.

13. Kumar K, et al. Novel selective thiadiazine DYRK1A inhibitor lead scaffold with human pancreatic $\beta$-cell proliferation activity. Eur J Med Chem. 2018;157:1005-1016.

14. Mussmann R, et al. Inhibition of GSK3 promotes replication and survival of pancreatic beta cells. J Biol Chem. 2007;282(16):12030-12037.

15. Liu H, et al. Glycogen synthase kinase-3 and mammalian target of rapamycin pathways contribute to DNA synthesis, cell cycle progression, and proliferation in human islets. Diabetes. 2009;58(3):663-672.

16. Annes JP, et al. Adenosine kinase inhibition selectively promotes rodent and porcine islet $\beta$-cell replication. Proc Natl Acad Sci USA. 2012;109(10):3915-3920.

17. Heit JJ, et al. Calcineurin/NFAT signalling regulates pancreatic beta-cell growth and function. Nature. 2006;443(7109):345-349

18. Demozay D, Tsunekawa S, Briaud I, Shah R, Rhodes CJ. Specific glucose-induced control of insulin receptor substrate-2 expression is mediated via Ca2+-dependent calcineurin/NFAT signaling in primary pancreatic islet $\beta$-cells. Diabetes. 2011;60(11):2892-2902.

19. Porat $\mathrm{S}$, et al. Control of pancreatic $\beta$ cell regeneration by glucose metabolism. Cell Metab. 2011;13(4):440-449.

20. Hille S, et al. Dyrk1a regulates the cardiomyocyte cell cycle via D-cyclin-dependent Rb/E2f-signalling. Cardiovasc Res. 2016;110(3):381-394.

21. Sadasivam S, DeCaprio JA. The DREAM complex: master coordinator of cell cycle-dependent gene expression. Nat Rev Cancer. 2013;13(8):585-595.

22. Frost D, et al. $\beta$-carboline compounds, including harmine, inhibit DYRK1A and tau phosphorylation at multiple Alzheimer's disease-related sites. PLoS ONE. 2011;6(5):e19264.

23. Wang $\mathrm{H}$, et al. Insights into beta cell regeneration for diabetes via integration of molecular landscapes in human insulinomas. Nat Commun. 2017;8(1):767.

24. Nica AC, et al. Cell-type, allelic, and genetic signatures in the human pancreatic beta cell transcriptome. Genome Res. 2013;23(9):1554-1562.

25. Blodgett DM, et al. Novel Observations From Next-Generation RNA Sequencing of Highly Purified Human Adult and Fetal Islet Cell Subsets. Diabetes. 2015;64(9):3172-3181.

26. Coutadeur S, et al. A novel DYRK1A (dual specificity tyrosine phosphorylation-regulated kinase 1A) inhibitor for the treatment 
of Alzheimer's disease: effect on Tau and amyloid pathologies in vitro. J Neurochem. 2015;133(3):440-451.

27. Ashford AL, et al. A novel DYRK1B inhibitor AZ191 demonstrates that DYRK1B acts independently of GSK3 $\beta$ to phosphorylate cyclin D1 at $\operatorname{Thr}(286)$, not $\operatorname{Thr}(288)$. Biochem J. 2014;457(1):43-56.

28. Zhang HH, Lipovsky AI, Dibble CC, Sahin M, Manning BD. S6K1 regulates GSK3 under conditions of mTOR-dependent feedback inhibition of Akt. Mol Cell. 2006;24(2):185-197.

29. Brissova M, Niland JC, Cravens J, Olack B, Sowinski J, Evans-Molina C. The Integrated Islet Distribution Program Answers the Call for Improved Human Islet Phenotyping and Reporting of Human Islet Characteristics in Research Articles. Diabetes. 2019;68(7):1363-1365.

30. Becker W, Sippl W. Activation, regulation, and inhibition of DYRK1A. FEBS J. 2011;278(2):246-256.

31. Debdab M, et al. Leucettines, a class of potent inhibitors of cdc2-like kinases and dual specificity, tyrosine phosphorylation regulated kinases derived from the marine sponge leucettamine B: modulation of alternative pre-RNA splicing. JMed Chem. 2011;54(12):4172-4186.

32. Ionescu A, Dufrasne F, Gelbcke M, Jabin I, Kiss R, Lamoral-Theys D. DYRK1A kinase inhibitors with emphasis on cancer. Mini Rev Med Chem. 2012;12(13):1315-1329.

33. Badura L, et al. An inhibitor of casein kinase I epsilon induces phase delays in circadian rhythms under free-running and entrained conditions. J Pharmacol Exp Ther. 2007;322(2):730-738.

34. Walton KM, et al. Selective inhibition of casein kinase 1 epsilon minimally alters circadian clock period. J Pharmacol Exp Ther. 2009;330(2):430-439.

35. Ring DB, et al. Selective glycogen synthase kinase 3 inhibitors potentiate insulin activation of glucose transport and utilization in vitro and in vivo. Diabetes. 2003;52(3):588-595.

36. Domínguez JM, Fuertes A, Orozco L, del Monte-Millán M, Delgado E, Medina M. Evidence for irreversible inhibition of glycogen synthase kinase-3 $\beta$ by tideglusib. J Biol Chem. 2012;287(2):893-904 